Abstract 106 Table 1 Distribution of outcome of VC, including: no further input required, blood test in primary care, cardiac test in hospital only, outpatient Cardiology clinic (OPA)

\begin{tabular}{|l|l|l|l|l|}
\hline & $\begin{array}{l}\text { No Further } \\
\text { Input }\end{array}$ & Blood Test Only & Cardiac Test Only & OPA \\
\hline $\begin{array}{l}\text { Primary Care 1 } \\
\text { (53 patient events) }\end{array}$ & $21(40 \%)$ & $3(6 \%)$ & $25(47 \%)$ & $4(7 \%)$ \\
\hline $\begin{array}{l}\text { Primary Care 2 } \\
\text { (74 patient events) }\end{array}$ & $32(43 \%)$ & $0(0 \%)$ & $32(43 \%)$ & $10(14 \%)$ \\
\hline Overall & $53(42 \%)$ & $3(2 \%)$ & $57(45 \%)$ & $14(11 \%)$ \\
\hline
\end{tabular}

clinics' (VC). A team of Cardiologists and General Practitioners (GP) collaborated to create a VC via a web-based video link, enabling discussion of patients being considered by primary care for referral to the Cardiology clinic. All available GPs were present to enable an educational aspect to the discussion, and the aim of the VC was to: (i) identify appropriate patients requiring OP review, (ii) enable early selection of required investigations, and (iii) identify patients not requiring further assessment. We aimed to assess the safety and efficacy of this approach.

Methods VC's were established between a Consultant Cardiologist and two primary care practices, and all referrals required an ECG. Each patient was presented by the referring GP, and the Consultant Cardiologist issued correspondence and co-ordinated any required tests and follow-up. A 13 month period of this practise was reviewed with 135 patient events screened via a cross-matched record of patients. Data was recorded for: indication for and outcome of discussion; likelihood patient would have had test or referral made without this service (as assessed by Cardiology); and whether patients discussed had a subsequent unplanned admission or cardiac assessment.

Results 8/135 were excluded (duplicate; admitted prior to VC; not discussed; or re-discussed for learning).

Efficacy Outcomes of the VC are summarised in table 1. The overall number of patients a GP 'would' have referred was 113 (89\%), and the number that 'would' have had tests booked 98 (77\%), across the two practises. This left an overall OPA post VC of $11 \%$ vs a potential $89 \%$ 'would' have been referred (100 potential appointments avoided), and an overall test rate post VC $48 \%$ vs $77 \%$ 'would' have had tests (37 potential tests avoided).

Safety Unplanned Cardiology input post-VC included 1 subsequent chest pain clinic, a different issue to the original VC discussion, and 1 admission with syncope, which was the issue that was discussed in VC but with no pathology identified during admission.

Conclusion We demonstrated a significant reduction in OP Cardiology appointments and hospital investigations, proving improved resource utilisation, and the associated safety data was reassuring. As a result, this service demonstrates a novel approach to technology-enabled streamlining of primary to secondary care services in a safe and efficient manner.

Conflict of Interest None

\section{NICE 2016 STABLE CHEST PAIN GUIDELINES: IMPROVED YIELD OF SEVERE CORONARY ARTERY DISEASE AT INVASIVE CORONARY ANGIOGRAPHY} ${ }^{1}$ Sarojini David, ${ }^{3}$ Antonis Pavlidis, ${ }^{4}$ Sven Plein, ${ }^{1}$ Khaled Alfakih. 'Lewisham \& Greenwich NHS Trust; ' ${ }^{2}$ niversity College London; ${ }^{3}$ Guy's and St. Thomas' NHS Foundation Trust; ${ }^{4}$ Institute of Cardiovascular and Metabolic Medicine, University of Leeds
Introduction The UK National Institute of Health and Care Excellence (NICE) updated its guidelines on management of chest pain in 2016. NICE now recommend CT coronary angiography (CTCA) as the first line investigation for all new patients presenting with stable chest pain. This is different from the NICE 2010 guidelines which recommended that patients with high pre-test probability of having coronary artery disease (CAD) are referred directly for invasive coronary angiography (ICA). We previously implemented the NICE 2010 guidelines and referred all patients with high probability of CAD for ICA, audited this practice and found a low yield of severe CAD of $30 \%(1)$. We have now implemented the NICE 2016 guideline in a district general hospital and evaluated rates of ICA and yield of severe CAD.

Methods We undertook a retrospective search of the local radiology database from January 2017 to June 2018. CTCA reports CAD grade as normal/minimal stenosis, mild (30$50 \%)$, moderate $(50-70 \%)$, or severe (>70\%). Subsequent downstream investigations were audited.

Results In total 652 patients underwent CTCA (mean age 55 yrs; 330 male). A total of 65 patients underwent ICA following an interpretable CTCA, with 41 patients found to have severe CAD, a yield of $63 \%$.

Importantly, 34 patients were found to have severe CAD on CTCA, with 30 attending for ICA which confirmed severe CAD in 22, a yield of $73 \%$. 18 patients went on to be revascularised.

58 patients were deemed to have moderate CAD on CTCA, with 37 referred for ICA of which 33 attended and 18 were found to have severe CAD. 18 were referred for imaging stress tests and only one had a positive test. The total yield of severe CAD for this subgroup at ICA was 55\%. 17 patients were revascularised.

Finally, a total of 63 patients were deemed to have mild disease, with 2 patients referred for ICA and one found to have severe CAD. The majority of patients $(n=462)$ had nor$\mathrm{mal} / \mathrm{minimal}$ CTCA stenosis. There were a total of 36 inconclusive CTCA studies.

Conclusion In patients undergoing ICA following CTCA, the overall yield of severe CAD was 63\%. This compares very well with our previous data when we applied the NICE 2010 guidelines, which recommended ICA as first line test for all patients with high likelihood of having CAD, wherein the yield of severe CAD was only $30 \%$.

The yield of severe CAD is likely to improve further with greater utilisation of imaging stress tests as a gatekeeper to ICA in patients with moderate stenosis at CTCA.

1.Rauf A DS, Pierres F, Jackson A, Papamichail N, Pavlidis A, Alfakih A. Current diagnostic yield of invasive coronary angiography at a district general hospital. $\mathrm{Br} \mathrm{J}$ Cardiol. 2017;24:(4).

Conflict of Interest None 